\title{
Moonlighting Protein Glyceraldehyde- 3-Phosphate Dehydrogenase: A Cellular Rapid-Response Molecule for Maintenance of Iron Homeostasis in Hypoxia
}

\author{
Himanshu Malhotra ${ }^{a}$ Manoj Kumar ${ }^{a}$ Anoop Singh Chauhana Asmita Dhiman ${ }^{a}$ \\ Surbhi Chaudhary ${ }^{\mathrm{a}}$ Anil Patidar ${ }^{\mathrm{a}}$ Pallavi Jaiswal ${ }^{\mathrm{a}}$ Kapil Sharma $^{\mathrm{a}}$ \\ Navdeep Sheokand ${ }^{a} \quad$ Chaaya lyengar Raje ${ }^{b} \quad$ Manoj Raje ${ }^{a}$ \\ anstitute of Microbial Technology, CSIR, Sector 39A, Chandigarh, India, bNational Institute of \\ Pharmaceutical Education \& Research, Punjab, India
}

\section{Key Words}

Hypoxia $\cdot$ Iron $•$ GAPDH $\cdot$ Rapid response

\begin{abstract}
:
Background/Aims: Hypoxia triggers a rapid increase in iron demand to meet the requirements of enhanced erythropoiesis. The mobilization of iron stores from macrophage to plasma as holo-transferrin (Tf) from where it is accessible to erythroid precursor cells impacts iron homeostasis. Despite the immediate need for enhanced iron uptake by bone marrow cells, numerous studies have shown that transferrin receptor levels do not rise until more than 24 hours after the onset of hypoxia, suggesting the existence of heretofore unknown rapid response cellular machinery for iron acquisition in the early stages of cellular hypoxia. Methods: We performed flow cytometry to measure cell surface levels of TfR1, GAPDH, and Tf binding after hypoxia treatment. We utilized FRET analysis and co-immunoprecipitation methods to establish the interaction between Tf and GAPDH. Results: In the current study, we demonstrated that hypoxia induces K562 cells to translocate the cytosolic moonlighting protein glyceraldehyde-3-phosphate dehydrogenase (GAPDH) onto cell surfaces and into the extracellular milieu to acquire transferrin-bound iron, even while levels of the classical transferrin receptor TfR1 (CD71) remain suppressed. GAPDH knockdown confirmed this protein's role in transferrin acquisition. Interestingly, macrophages did not show enhanced levels of extracellular GAPDH under hypoxia. Conclusion: Our results suggest the role of GAPDH-mediated Tf uptake as a rapid response mechanism by which cells acquire iron during the early stages of hypoxia. This is a tissue-specific phenomenon for the distinct requirements of cells that are consumers of iron versus cells that play a role in iron storage and recycling. This rapid deployment of an abundantly available multipurpose molecule allows hypoxic cells
\end{abstract}

\begin{tabular}{ll}
\hline Dr. Manoj Raje & Institute of Microbial Technology, CSIR \\
& Sector 39A, Chandigarh (India) \\
& Tel. +91-172-6665198, Fax +91-172-2690632, E-Mail manoj@imtech.res.in
\end{tabular}




\section{Cellular Physiology Cell Physiol Biochem 2019;52:517-531 \\ \begin{tabular}{ll|l} 
DOl: 10.33594/000000037 & $\begin{array}{l}\text { O 2019 The Author(s). Published by } \\
\text { Cell Physiol Biochem Press GmbH\&Co. KG }\end{array}$
\end{tabular} \\ Malhotra et al.: GAPDH-a Rapid Responder for Maintaining Iron Homeostasis in \\ Hypoxia}

to internalize more Tf and maintain enhanced iron supplies in the early stages of hypoxia before specialized receptors can be synthesized and deployed to the cell membrane.

(C) 2019 The Author(s). Published by Cell Physiol Biochem Press GmbH\&Co. KG

\section{Introduction}

Iron is an essential element for every form of life. It comprises an integral part of heme which is crucial for supplying oxygen to cells and tissues, thereby conferring a close association between oxygen delivery and iron availability [1]. In the human body, the majority of available iron is utilized in erythropoiesis which is enhanced during hypoxia. This causes an increased number of RBCs to be released into circulation, thereby enhancing oxygen delivery capacity. A prerequisite for this activity is an increase in iron delivery to erythrocyte precursor cells [2]. In serum, iron remains bound to transferrin, an abundant iron transport protein with high affinity for iron. Cellular iron uptake occurs chiefly via receptor-mediated uptake of holo-transferrin by two well-documented Tf receptors-TfR1 and TfR2. TfR1 is a high affinity ubiquitously expressed receptor, while the expression of TfR2 is restricted to certain cell types and is unaffected by intracellular iron concentrations [3, 4]. Hypoxiatreated cells enhance their iron uptake by increasing the capture and internalization of Tf [5].

Acute hypoxia induces a rapid mobilization of iron from storage cells into plasma to ensure adequate iron supplies to maturing red blood cells for proper hemoglobinization [6]. An increase in erythropoietin (EPO) mRNA and plasma EPO concentration within 90 mins to $6 \mathrm{hrs}$ of hypoxic exposure stimulates reticulocyte proliferation and maturation $[7,8]$. This requires a concurrent rapid increase in cellular iron acquisition. The immediate enhanced requirement for iron under hypoxia is not only necessary for the synthesis of heme to be incorporated into hemoglobin but also for the protection of cells from reactive oxygen species (ROS) that may accumulate as a consequence of cellular oxygen deprivation. Hypoxia is known to enhance ROS generation for the activation of HIF- $1 \alpha$ to counter hypoxia $[9,10]$. ROS are also known to cause cellular damage [11] and the mobilization of enzymes such as catalase (which requires iron) is necessary, as they are key components of the cellular antioxidant defense machinery $[12,13]$.

Despite the pressing need for immediate access to higher levels of iron, increased mRNA levels of TfR1 are observed only after $16 \mathrm{hrs}$ of hypoxia [14]. Other in vitro studies of acute hypoxia utilizing several cellular model systems have also confirmed that levels of TfR 1 at the cell surface remain unchanged for more than 24 hours, despite the increased capture of Tf by the cells in the interim period $[15,16]$. This anomalous situation in the face of hypoxia can only be explained by the existence of alternative rapid-response Tf-acquisition machinery that can be rapidly deployed before TfR1 expression on the cell surface is upregulated. Abundantly and constitutively expressed moonlighting proteins would be prime candidates that could be rapidly repurposed in a short time frame for such emergency situations before protracted homeostatic mechanisms allow enhanced synthesis and deployment of specialized molecules. Previous studies in a diverse range of prokaryotic and eukaryotic cells have revealed that the multifunctional glycolytic enzyme GAPDH functions as an evolutionarily-conserved cell surface receptor for the iron carrier proteins transferrin and lactoferrin. Upon iron starvation, many cells prefer the use of rapidly externalized GAPDH for iron restitution [17-22]. Earlier, we demonstrated that cells enhance their secretion of GAPDH into the extracellular milieu (sGAPDH) when they are depleted of iron. This sGAPDH functions as an autocrine and/or paracrine receptor that traffics iron-replete transferrin and lactoferrin into cells to restore intracellular iron levels $[23,24]$. The detailed biochemical and biophysical characteristics regarding the interaction between transferrin and GAPDH (recruited to the cell membrane and secreted from cells upon iron starvation) and the endocytosis of transferrin by GAPDH have been described earlier [17, 24]. As hypoxia and 


\section{Cellular Physiology Cell Physiol Biochem 2019;52:517-531

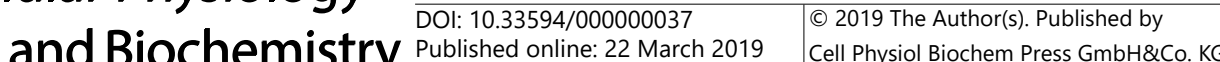 \\ Malhotra et al.: GAPDH-a Rapid Responder for Maintaining Iron Homeostasis in \\ Hypoxia}

iron deprivation impart similar physiological consequences, we explored the possibility that GAPDH plays the role of a rapid response molecule for iron trafficking in the early stages of acute cellular hypoxia.

\section{Materials and Methods}

\section{Cell lines and cell culture}

K562 (human chronic myelogenous leukemia cell line) and J774 (mouse macrophage cell line) were obtained from the National Centre for Cell Sciences (NCCS), India and the European Collection of Authenticated Cell Cultures (ECACC), UK, respectively. The CHO-TRVb cell line, a variant of the CHO cell line which lacks both classical transferrin receptors (TfR1 and TfR2) but can acquire transferrin via GAPDH [17, 24, 25], was a kind gift from Prof. T. McGraw (Weill Cornell Medical College, New York, NY, USA) and Dr. S. Mayor (NCBS, Bangalore, India). K562 and J774 cells were maintained in RPMI medium and DMEM

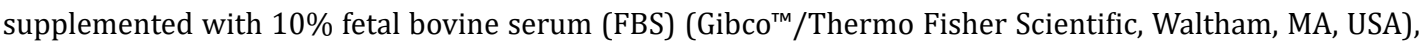
respectively. Peritoneal macrophages were obtained from 6-8-week-old Balb/c mice that were euthanized by cervical dislocation according to methods described previously [26].

\section{GAPDH silencing and activity assays}

For cellular GAPDH knockdown, K562 cells were transfected with human GAPDH shRNA lentiviral particles (Sigma-Aldrich, St. Louis, MO, USA) according to the manufacturer's instructions. Stably transfected cells were cultured in medium supplemented with puromycin for selection pressure $(12 \mu \mathrm{g} /$ $\mathrm{mL}$ ). Knockdown of GAPDH was confirmed by a GAPDH activity assay using a KDalert ${ }^{\mathrm{TM}}$ GAPDH assay kit (Ambion ${ }^{\circledR} /$ Thermo Fisher Scientific) according to the manufacturer's instructions.

\section{Induction of hypoxia in cells}

Cells in culture were exposed to hypoxia for $24 \mathrm{hrs}$ using a New Brunswick ${ }^{\mathrm{TM}}$ Galaxy® $48 \mathrm{R}$ incubator (Eppendorf, Hamburg, Germany). The oxygen level was kept at $1 \%$ using nitrogen along with $5 \% \mathrm{CO}_{2}$. Control cells were kept in a normal incubator with $5 \% \mathrm{CO}_{2}$. $\mathrm{K} 562$ cells were placed into the hypoxic chamber immediately, while J774, CHO-TRVb, and peritoneal macrophages were first allowed to adhere overnight in tissue culture plates before being subjected to hypoxia treatment. After exposure to hypoxia, cell viability was assessed by multiple independent assays. These included: (i) trypan blue exclusion assay; (ii) MTT assay using an EZcount ${ }^{\mathrm{TM}}$ MTT cell assay kit (HIMEDIA ${ }^{\oplus}$, Mumbai, India); (iii) AnnexinV-7AAD [BD Biosciences (San Jose, CA, USA) and Invitrogen/Thermo Fisher Scientific, respectively] co-staining coupled with flow cytometry (FACS); and (iv) caspase-3/7 activation using Caspase-Glo ${ }^{\circledR} 3 / 7$ assay kit (Promega, Madison, WI, USA). Typically, the cell viability of hypoxia-treated cells was $>95 \%$.

\section{Analysis of GAPDH and CD71 mRNA by quantitative reverse transcription}

Total RNA was isolated from control and hypoxia-treated cells using an RNA isolation kit (Promega) then subjected to reverse transcription using a QuantiTect $®$ reverse transcription kit (Qiagen, Hilden, Germany). To confirm the amplification of equal amounts of cDNA, control PCR reactions with primers specific for tubulin were run in parallel. Real-time PCR was performed with a QuantiFast SYBR® Green RT-PCR kit (Qiagen). All kits were utilized according to the manufacturers' protocols. For human-TfR1 amplification, the forward and reverse primer sequences were 5'-CTA TGA GAG GTA CAA CAG CC-3' and 5'-GTC AAT GTC GTC CCA AAC GTC AC-3', respectively [27]. For tubulin controls, the forward and reverse primer sequences were 5'-GGA GGA TGC TGC CAA TAA CT-3' and 5'-GGT GGT GAG GAT GGA ATT GT-3', respectively [28]. C $_{\mathrm{T}}$ values were calculated for each gene under control and hypoxia conditions. Fold-changes in mRNA levels in hypoxia-treated cells compared to control cells were calculated using the Livak method [29].

\section{Flow cytometry analysis}

Cell surface expression of GAPDH and CD71 (TfR1) and transferrin binding were evaluated by flow cytometry using $5 \times 10^{5}$ cells for each assay as described previously $[17,26]$. Briefly, after washing cells with FACS buffer (5\% FBS, $20 \mathrm{mM}$ HEPES, $150 \mathrm{mM} \mathrm{NaCl}, 5 \mathrm{mM} \mathrm{KCl} \mathrm{2}_{2}, 1 \mathrm{mM} \mathrm{CaCl} \mathrm{C}_{2}$ and $1 \mathrm{mM} \mathrm{MgCl}{ }_{2}$ ), cells were blocked using FACS block (FACS buffer supplemented with $5 \%$ each of normal human serum and normal goat 


\section{Cellular Physiology Cell Physiol Biochem 2019;52:517-531 \\ \begin{tabular}{ll|l} 
and Binch $10.33594 / 000000037$ & C) 2019 The Author(s). Published by
\end{tabular} \\ and BIOChemistry Published online: 22 March 2019 Cell Physiol Biochem Press GmbH\&Co. KG \\ Hypoxia}

serum) at $4{ }^{\circ} \mathrm{C}$ for 30 mins. Subsequently, samples were incubated for $1 \mathrm{hr}$ at $4{ }^{\circ} \mathrm{C}$ with specific antibodies. To evaluate surface GAPDH expression, $1 \mu \mathrm{g}$ of rabbit anti GAPDH (Sigma-Aldrich) or isotype control [rabbit IgG (Bangalore Genei, Bangalore, India)] was used for primary staining. Subsequently, cells were washed with FACS buffer and bound anti-GAPDH antibody was detected using anti rabbit-Alexa Fluor® 647 or antirabbit FITC. Surface CD71 expression was evaluated by directly incubating blocked cells with $1 \mu \mathrm{L}$ of antiCD71 PE/APC/FITC or matched isotype control (BD Biosciences or eBioscience/Thermo Fisher Scientific). The antibody used was either anti-human (for K562 cells) or anti-mouse (for J774 or mouse peritoneal macrophages). Total transferrin binding was assessed by incubating cells with $10 \mu \mathrm{g}$ of Tf-Alexa Fluorß 647/Tf-Alexa Fluor® 633 (Invitrogen/Thermo Fisher Scientific). All cell samples were counter-stained with 7-AAD (Invitrogen/Thermo Fisher Scientific). Fluorescence intensity data from $1 \times 10^{4}$ cells/sample were acquired and analyzed using FACS Verse or BD Accuri flow cytometers (BD Biosciences). Signal from dead cells was excluded by gating out the 7-AAD positive cells [30]. Data was collected from $10^{4}$ cells for each sample and all experiments were repeated three times. Results for staining intensity are presented as mean fluorescence intensity \pm standard error of the mean (MFI \pm SEM).

Flow cytometry-based measurement of GAPDH partitioning into detergent-resistant and detergentsoluble domains of plasma membrane

Analysis of the partitioning of GAPDH into detergent-resistant and detergent-soluble domains of the cell membrane was performed as described in previous protocols utilizing fluorescent antibody labeling coupled with flow cytometry [17, 31]. Hypoxia-exposed and control K562 cells were washed with FACS buffer and stained with anti-GAPDH antibody as described previously. After three washes with FACS buffer, one aliquot comprising of half of each set of cell samples was treated with $0.2 \%$ Triton X-100 at $25^{\circ} \mathrm{C}$ for 5 mins and analyzed by flow cytometry to quantify the amount of GAPDH retained in the membrane after detergent extraction as compared to normoxic cells. An increase in the flow cytometric differential detergent resistant ratio (FCDR ratio, which is calculated as: mean fluorescent signal of target molecules in detergent-resistant membrane fraction (DRM) / mean fluorescence signal of target molecules on whole cell membrane) is indicative of preferential partitioning of the target antigen (GAPDH) in the detergentresistant membrane domains [31].

\section{Uptake kinetics of transferrin}

Aliquots of $5 \times 10^{5}$ cells (treated and control cells) were first blocked with $2 \%$ BSA for 30 mins at $4{ }^{\circ} \mathrm{C}$ then incubated with $10 \mu \mathrm{g}$ of Tf-A633 in $200 \mu \mathrm{L}$ of pre-warmed serum-free medium (SFM) for different time points $(0,1,2,3,4,5,7.5,10,12.5,15,20,25$, and $30 \mathrm{mins})$. Internalization was stopped by the addition of excess chilled SFM and shifting the tubes onto ice. After two washes with SFM, cells were treated with $0.1 \%$ pronase to remove any transferrin that had not been internalized and was still retained on the cell surface. Intracellular $\mathrm{Tf}$ (which escaped pronase digestion) was measured by flow cytometry [17].

\section{Co-localization of GAPDH with transferrin and membrane raft marker cholera toxin}

For co-localization studies, hypoxia-treated K562 cells were first blocked with FACS block for 30 mins at $4{ }^{\circ} \mathrm{C}$. Subsequently, cells were incubated with rabbit anti-GAPDH antibody ( $1 \mu \mathrm{g}$ ) in SFM for $1 \mathrm{hr}$ at $4{ }^{\circ} \mathrm{C}$ then washed three times with SFM. Samples were then incubated for $1 \mathrm{hr}$ at $4{ }^{\circ} \mathrm{C}$ with a combination of antirabbit FITC (Sigma-Aldrich), $10 \mu \mathrm{g}$ of Tf-A546, and $1 \mu \mathrm{g}$ of CTX-A647 before shifting to $37{ }^{\circ} \mathrm{C}$ for 15 mins. After washing with chilled SFM, cells were fixed with $4 \%$ paraformaldehyde, mounted on coverslips, and imaged with a Nikon A1R confocal microscope (Nikon, Tokyo, Japan) using a 60× oil immersion objective with a pinhole aperture of 1 airy unit (AU).

\section{FRET analysis}

The interaction of transferrin and cell surface GAPDH that had co-internalized into endosomes was confirmed by acceptor photobleaching förster resonance energy transfer (FRET) analysis. K562 cells were first blocked with FACS block for 30 mins at $4{ }^{\circ} \mathrm{C}$ then incubated with rabbit anti-GAPDH antibody $(1 \mu \mathrm{g})$ in SFM for $1 \mathrm{hr}$ at $4{ }^{\circ} \mathrm{C}$ and washed 3 times with SFM. Subsequently, cells were incubated with anti-rabbit Alexa Fluor $₫ 568$ (donor) and $10 \mu$ g of Tf-Alexa Fluor $® 633$ (acceptor) for 1 hr at $4{ }^{\circ} \mathrm{C}$ then shifted to $37^{\circ} \mathrm{C}$ for 15 mins. After washing with chilled SFM, cells were fixed with 4\% paraformaldehyde and processed for microscopy. A control with goat IgG-A633 (unrelated protein) instead of Tf-A633 was analyzed in parallel. 


\section{Cellular Physiology Cell Physiol Biochem 2019;52:517-531

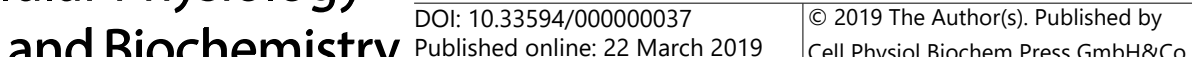

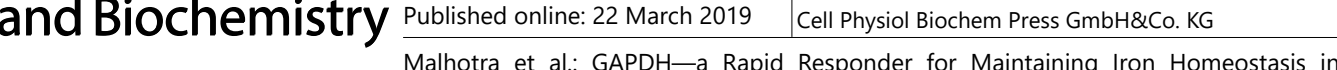 \\ Hypoxia}

FRET experiments were carried out using the acceptor photobleaching method [32]. FRET efficiency was calculated using the formula: FRET efficiency $=\{[$ Donor intensity (postbleach)-Donor intensity (prebleach) $]$ / Donor intensity (postbleach)\} S x 100.

\section{Co-immunoprecipitation of GAPDH-transferrin from plasma membrane surface}

Post hypoxia treatment, $5 \times 10^{7} \mathrm{~K} 562$ cells were washed with PBS and blocked with $2 \%$ BSA in SFM for 30 mins at $4{ }^{\circ} \mathrm{C}$. Subsequently, cells were incubated with $1 \mathrm{~mL}$ of $1 \mathrm{mg} / \mathrm{mL}$ biotinylated holo-transferrin (SigmaAldrich) in SFM at $4{ }^{\circ} \mathrm{C}$ for $1 \mathrm{hr}$, washed three times with SFM, and resuspended in $1 \mathrm{~mL}$ of homogenization buffer (20 mM Tris-HCl pH 7.4, 1 mM EDTA, 0.25 M sucrose) supplemented with protease inhibitor cocktail (Thermo Fisher Scientific) and $1 \mathrm{mM}$ PMSF. As a negative control, biotinylated transferrin was omitted in an aliquot of cells processed in parallel. Subsequently, the plasma membrane protein fraction was prepared as described previously [26]. Co-immunoprecipitation (Co-IP) was carried out using streptavidin magnetic beads (Pierce ${ }^{\mathrm{TM}} /$ Thermo Fisher Scientific) according to the manufacturer's instructions. After Tf pull-down, beads were boiled in SDS sample buffer and the co-precipitated proteins were analyzed via western blotting as described below.

\section{Western blot analysis}

Cell membrane fractions, whole cell lysate, or co-immunoprecipitated samples were resolved using $10 \%$ SDS-PAGE. For cell lysate and membrane fraction blots, $40 \mu \mathrm{g}$ and $100 \mu \mathrm{g}$ of protein was loaded, respectively. The separated proteins were then transblotted onto nitrocellulose membranes and the membranes were blocked using $2 \%$ casein in PBS $+0.05 \%$ Tween-20 (PBST). Blots were then probed overnight at $4{ }^{\circ} \mathrm{C}$ with monoclonal mouse anti-GAPDH antibody $(1 \mu \mathrm{g} / \mathrm{mL}$ ) (Calbiochem, San Diego, CA, USA), rabbit anti TfR2 (1 $\mu \mathrm{g} / \mathrm{mL}$ ) (Abcam, Cambridge, UK), or rabbit-anti HIF-1 $\alpha$ (Santa Cruz Biotechnology, Dallas, TX, USA). Bound antibodies were then detected using anti-mouse peroxidase or anti-rabbit peroxidase antibodies (SigmaAldrich), respectively. After extensive washing with PBST, blots were developed using Luminata ${ }^{\mathrm{TM}}$ Forte Western HRP substrate (MilliporeSigma, Burlington, MA, USA). As a loading control, anti beta-actin was used for cell lysates. For membrane fractions, amido black staining was utilized to ensure equal protein loading. Blots were imaged on an ImageQuant LAS 4000 imager (GE Healthcare Life Sciences, Marlborough, MA, USA).

\section{Cellular iron levels}

The total iron content of cells was estimated as previously described [33]. Briefly, $1 \times 10^{7}$ control or hypoxia-treated cells were harvested and washed with neutral buffer. To release iron, cells pellets were digested in $5 \% \mathrm{HNO}_{3}$ at $80{ }^{\circ} \mathrm{C}$ for $2 \mathrm{hrs}$. Digested samples were centrifuged and the supernatant was used for iron estimation using a Quantichrom iron assay kit (Bioassay Systems, Hayward, CA, USA) according to the manufacturer's instructions. The intracellular labile iron pool was assessed by measuring the quenching of calcein fluorescence as described previously [32]. After $24 \mathrm{hr}$ of hypoxia treatment, cells were harvested, washed three times with SFM, and incubated in $500 \mathrm{nM}$ Calcein-AM (Invitrogen/Thermo Fisher Scientific) at $37^{\circ} \mathrm{C}$ for $10 \mathrm{~min}$. Excess dye was removed by extensive washing with SFM. The fluorescence signal of intracellular Calcein-AM was measured by flow cytometry.

\section{Catalase assay}

Catalase activity was measured in control and hypoxic cells as described previously [34]. Briefly, $2 \times 10^{6} \mathrm{~K} 562$ cells exposed to hypoxia for $24 \mathrm{hrs}$, were washed with PBS and gently lysed in $100 \mu \mathrm{L}$ of ice-cold 1\% Triton X-100. The lysate was centrifuged at $14000 \times g$ in a pre-cooled centrifuge for 10 mins. Supernatant (50 $\mu$ g protein) was added to a $10 \mathrm{~mL}$ solution of $10 \mathrm{mM} \mathrm{H}_{2} \mathrm{O}_{2}$ in $50 \mathrm{mM}$ phosphate buffer ( $\mathrm{pH}$ 7.0). The disappearance of $\mathrm{H}_{2} \mathrm{O}_{2}$ was immediately measured as a decrease in absorbance (extinction coefficient $=36 \mathrm{~m}^{-1} \mathrm{~cm}^{-1}$ at $240 \mathrm{~nm}$ ) within $1 \mathrm{~min}$. A set of control (normoxic cells) was run in parallel for comparison. Catalase activity was expressed in units/milligrams of protein $/ \mathrm{min}$. The increase in catalase activity under hypoxia was presented as \% of control (normoxic cells cultured in parallel). 


\section{Cellular Physiology Cell Physiol Biochem 2019;52:517-531 \\ \begin{tabular}{ll|l} 
and Binc $10.33594 / 000000037$ & (c) 2019 The Author(s). Published by
\end{tabular} \\ Published online: 22 March 2019 Cell Physiol Biochem Press GmbH\&Co. KG \\ Malhotra et al.: GAPDH—a Rapid Responder for Maintaining Iron Homeostasis in \\ Hypoxia}

Extracellular secretion of GAPDH and its role in transferrin uptake

Media supernatant from hypoxia treated and control K562 cells ( $5 \times 10^{5}$ cells/assay) were collected and centrifuged at $500 \mathrm{~g}$ for 5 mins to remove debris. GAPDH content of the clear supernatant was measured using a GAPDH Assay Kit (Ambion®/Thermo Fisher Scientific) as described above. These supernatants were labeled as control-medium supernatant and hypoxia-medium supernatant, respectively. To evaluate the role of this secreted GAPDH in transferrin acquisition by cells, $5 \times 10^{5}$ cells were incubated with $200 \mu \mathrm{L}$ of respective media supernatant along with $10 \mu \mathrm{g}$ of TF-A647 for $30 \mathrm{mins}$ at $37^{\circ} \mathrm{C}$ then treated with pronase as described above. The internalized transferrin signal was analyzed by flow cytometry using a BD FACS Verse instrument (BD Biosciences) [24].

\section{Statistical analysis}

Statistical analysis was performed using Student's unpaired T-test.

\section{Results}

Modulation of cell surface CD71 (TfR1), transferrin binding, and internalization upon hypoxia exposure

At the tissue level, hypoxia results in a rapid demand for iron so as to produce more erythrocytes in bone marrow. For this study, we chose the K562 cell line as the primary model of study along with the CHO-TRVb cell line, the cell surfaces of which lack TfR1 and TfR2. Hypoxia is known to enhance the expression of HIF-1 $\alpha$ and upregulate enzymes involved in glycolysis. In the case of GAPDH, hypoxia is also known to upregulate this protein for alternate functions that are not associated with glycolysis [35, 36]. We first confirmed the induction of a hypoxic response in K562 cells by observing an increase in the expression of HIF- $1 \alpha$ and total cellular GAPDH activity (Fig. 1A \& B), which is consistent with earlier reports by other researchers [16]. TfR1 is regulated both at transcription and post-transcriptionally by HIF-1a and IRP-1. It had been shown previously that hypoxia causes an increase in TfR1 mRNA levels after 16 hrs of hypoxic exposure [5, 14]; we also obtained similar results (Fig. 1C). Though TfR1 mRNA starts to increase soon after subjecting cells to hypoxia, several investigators have reported that at the protein level, cell surface expression of TfR1 remains unchanged or below normal for more than 24 hrs after hypoxic exposure [15, 16, 37]. One study even reported $11 \%$ reduction in cellular TfR 1 levels after 24 hrs of hypoxia exposure in lens epithelial cells [38]. Another study utilizing HEK293 cells found that the increase in the levels of TfR mRNA during hypoxia does not cause any significant change in the amount of TfR protein; the investigators attempted to explain that the observed contradictory increase in cellular iron resulted from alternate unknown mechanisms [39]. Confirming this apparent disconnect between mRNA and protein levels, we also found a significant decrease in the expression of CD71 on the surface of hypoxic K562 cells (Fig. 1D). Despite this decrease in CD71, transferrin binding to both K562 and CHO-TRVb (which do not express TfR1) cells was significantly higher (Fig. 1E \& F). This suggests the existence of an additional uncharacterized transferrin receptor. Our results are similar to the findings of Toth et al. who demonstrated a greater than two-fold increase in surface binding of Tf early-on during hypoxia in K562 and Hep3B cells without additional TfR1 expression [5]. Cell internalization assays revealed that the increased Tf binding by hypoxic K562 cells is also accompanied by enhanced endocytosis of the ligand in a time-dependent manner when compared to control cells (Fig. 1G). K562 cells are also known to express TfR2. However, unlike TfR1, TfR2 is not modulated by cellular iron levels. Though it has been shown to internalize transferrin, TfR2 is unable to compensate for the absence of TfR1 and its role in iron acquisition is not yet fully understood. Calzolari et al. have reported that exposure to hypoxia can cause a decrease but not an increase in TfR2 levels [40, 41]. Upon subjecting K562 cells to hypoxia, we found that both cellular and plasma membrane levels of TfR2 remained unchanged (Fig. 1H \& I), suggesting that TfR2 may not play a significant role in additional transferrin acquisition during hypoxia. 


\section{Cellular Physiology Cell Physiol Biochem 2019;52:517-531 \\ \begin{tabular}{ll|l} 
and Biochemisty $10.33594 / 000000037$ & C 2019 The Author(s). Published by \\
Published online: 22 March 2019 & Cell Physiol Biochem Press GmbH\&Co. KG
\end{tabular} \\ Malhotra et al.: GAPDH—a Rapid Responder for Maintaining Iron Homeostasis in \\ Hypoxia}

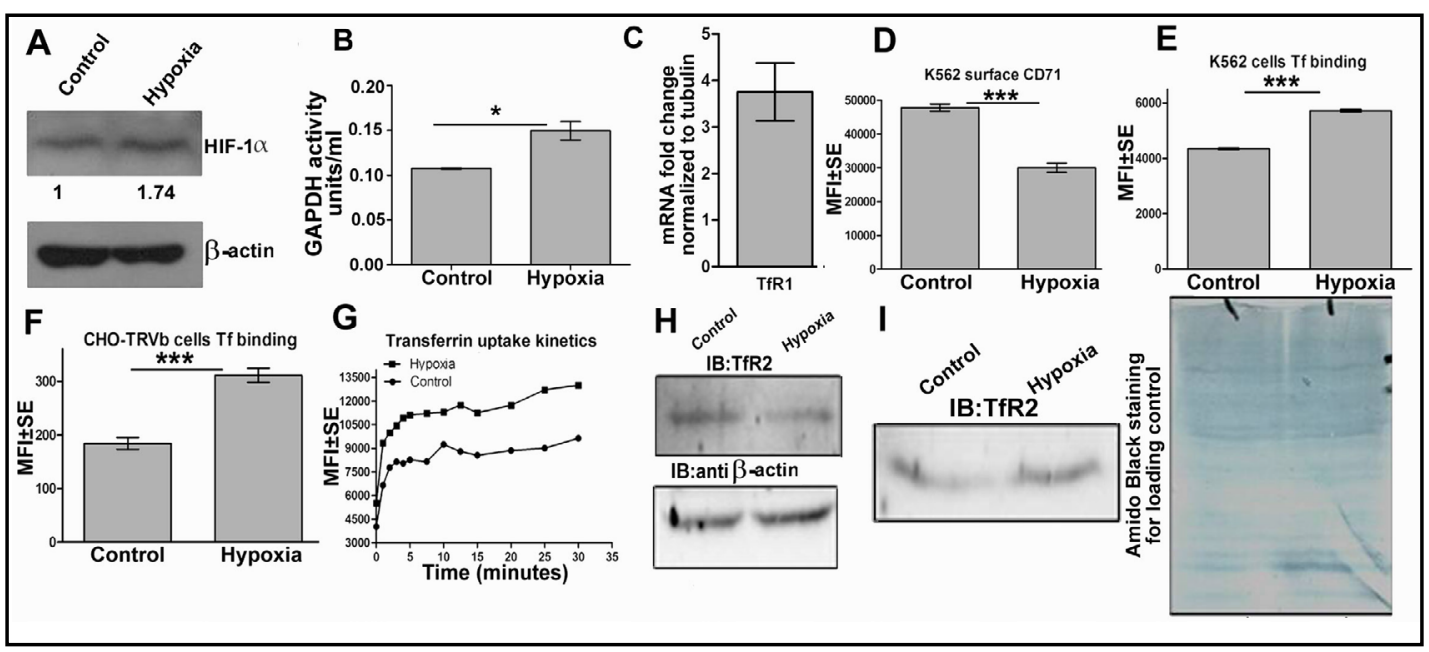

Fig. 1. TfR1 and TfR2 are not involved in enhanced Tf binding and uptake in early stages of hypoxia. (A) HIF-1 $\alpha$ expression is enhanced after exposure to low oxygen levels. Cell lysate prepared from 1x106 K562 cells, cultured for $24 \mathrm{hrs}$ in hypoxic or normoxic conditions. The Western blot was probed with rabbit HIF-1 $\alpha$ antibody. Equal loading was confirmed by staining with monoclonal mouse $ß$-actin antibody. Fold change was assayed using Scion image software. IB=immunoblotting. (B) Hypoxia treated cells express enhanced GAPDH. Control and 24 hrs hypoxia treated K562 cells were analyzed for their total GAPDH activity, p $<0.05, \mathrm{n}=2$. (C) Analysis of CD71 mRNA by Real time PCR revealed $\sim 4$ fold increase in TfR1 mRNA, p<0.001, $\mathrm{n}=3$, repeated twice. (D) K562 cells exposed to $24 \mathrm{hrs}$ of hypoxia were stained for surface expression of TfR1 (CD71) and evaluated by flow cytometry. A significant reduction in cell surface expression of TfR1 as compared to control cells is evident, $\mathrm{p}<0.0001, \mathrm{n}=104$ cells for each sample, all experiments were repeated three times. (E\&F) In spite of decreased TfR1 expression the total transferrin binding to cells is significantly enhanced by hypoxia. K562 and CHO-TRVb cells exposed to $24 \mathrm{hrs}$ of hypoxia were assayed for transferrin binding by flow cytometry, $\mathrm{p}<0.0001, \mathrm{n}=104$. (G) Besides increase in cell surface capture, hypoxia also causes enhanced transferrin internalization by cells. Data is presented as change in mean fluorescence intensity \pm SE as a function of time, for each time point $n=104$ cells. Even after 24 hrs of hypoxia cellular TfR2 levels remain unchanged: Total cell lysate $(\mathrm{H})$ and membrane fraction (I) of K562 cells was prepared and run on $10 \%$ SDS PAGE, transferred to nitrocellulose membrane and probed for TfR2 using rabbit antihuman TfR2. Equal loading was confirmed by immunostaining actin (lysates) and amido black staining (membrane fractions).

Hypoxia enhances cell surface expression of GAPDH in plasma membrane raft domains which binds and internalizes holo-Tf to deliver iron into cells

The significant decrease in CD71 or TfR1 cannot explain enhanced Tf binding and internalization. We therefore proceeded to investigate cell surface-GAPDH expression, given that this protein had earlier been demonstrated to moonlight as a transferrin receptor in iron-starved cells $[17,26]$. A significant enhancement in the expression of GAPDH on the surface of hypoxia-treated K562 and CHO-TRVb cells was noted (Fig. 2A \& B). Since GAPDH that partitions into the detergent-resistant fraction of the plasma membrane has been shown to preferentially capture holo-transferrin, [42] we examined the localization of the GAPDH deployed to the plasma membrane upon hypoxic exposure. Interestingly, in comparison to control normoxic cells, hypoxic cells demonstrated a significant increase in the FCDR ratio (the fraction of membrane GAPDH that is resistant to extraction by non-ionic detergent) (Fig. 2C \& D). This indicates that the newly-deployed GAPDH receptor is similar to the GAPDH recruited on the cell membrane during iron starvation. Therefore, this cell surface GAPDH may also be involved in the acquisition of iron-bound transferrin. After observing that transferrin binding and uptake increased along with a correlated enhancement of surface GAPDH expression in hypoxia-treated cells, we decided to investigate whether the two molecules interact and cotraffic into cells. This involved investigating the association between surface internalized 


\section{Cellular Physiology Cell Physiol Biochem 2019;52:517-531 \\ \begin{tabular}{ll|l} 
and Biochemistry & $\begin{array}{l}\text { DOl: 10.33594/000000037 } \\
\text { Published online: } 22 \text { March } 2019\end{array}$ & $\begin{array}{l}\text { O } 2019 \text { The Author(s). Published by } \\
\text { Cell Physiol Biochem Press GmbH\&Co. KG }\end{array}$ \\
\cline { 2 - 3 } & Men
\end{tabular} \\ Malhotra et al.: GAPDH-a Rapid Responder for Maintaining Iron Homeostasis in \\ Hypoxia}

GAPDH with Tf in K562 cells treated with hypoxia. Utilizing confocal microscopy, GAPDH was observed to co-localize with Tf and the raft marker (membrane rafts represent DRM) cholera toxin at the cell surface and also within endosomes (Fig. 3A). To further validate our results, acceptor photobleaching-based FRET (Förster resonance energy transfer) analysis was carried out to verify the interaction of GAPDH internalized from the surface of the hypoxic K562 cells along with transferrin into endosomes (Fig. 3B \& C). Finally, we performed a co-immunoprecipitation assay from a membrane fraction of hypoxic K562 cells utilizing biotinylated transferrin. A pull-down of transferrin confirmed its interaction with GAPDH on the surface of hypoxic K562 cells (Fig. 3D). Given that we had observed an increase in trafficking of transferrin into hypoxic cells, we compared the total cellular iron levels of control and hypoxia-exposed cells. We found a significant increase in the iron content of both K562 and CHO-TRVb cells exposed to hypoxia (Fig. 3E \& F). This confirms that the alternate transferrin iron acquisition machinery is able to ameliorate the effects of decreased TfR1. One of the most common immediate destinations for iron imported into hypoxic cells is its incorporation into catalase. This iron-dependent enzyme is required for the neutralization of $\mathrm{H}_{2} \mathrm{O}_{2}$ that accumulates as a consequence of hypoxia-mediated ROS formation. We assessed the level of catalase in cells subjected to hypoxia and observed a significant enhancement of enzymatic activity (Fig. 3G). This result is corroborated by previous studies demonstrating that hypoxia causes an increase in rat erythrocyte catalase activity [12].

Knockdown-based analysis confirms the role of GAPDH in enhanced transferrin-mediated iron acquisition by hypoxic cells

A GAPDH-knockdown K562 cell line was generated with 78\% knockdown of total cellular GAPDH. Unlike wild-type cells, there was no significant increase in surface GAPDH expression upon hypoxia treatment (Fig. 4A and inset); however, similar to wild- type cells, GAPDH K/D cells also demonstrated a significant decrease in surface CD71 expression upon exposure to hypoxia (Fig. 4B). GAPDH K/D cells demonstrated significantly less transferrin binding upon treatment with hypoxia (Fig. 4C). This was unlike the enhanced transferrin binding observed in hypoxia-treated wild-type K562 cells (Fig. 1E). The decrease in transferrin binding in GAPDH K/D cells was also accompanied with lower total iron content (Fig. 4D). This suggests that the newly-deployed iron acquisition machinery was compromised. These results confirm a role for GAPDH as an additional transferrin receptor for iron acquisition under hypoxia.

GAPDH secretion is enhanced under hypoxia and the culture supernatant of hypoxiatreated cells enhances transferrin uptake

Previous research demonstrated that GAPDH is actively secreted out of cells (sGAPDH) where it associates with transferrin and lactoferrin as a soluble receptor and effects an autocrine and paracrine delivery of iron into cells especially during conditions of cellular stress $[23,24]$. To investigate the possible role of secreted GAPDH in Tf acquisition during hypoxic stress, we investigated and noted a significant increase in the secretion of GAPDH into the culture supernatant upon exposure of K562 cells to hypoxia (Fig. 4E). When utilized for evaluating Tf uptake by cells, the hypoxic cell supernatant demonstrated a significant increase in Tf delivery compared to the supernatant from normoxic cells (Fig. 4F). This is similar to our earlier observation involving enhanced delivery of transferrin and associated iron into cells that are incubated with culture supernatant obtained from iron-starved cells that also contains elevated levels of secreted GAPDH [24]. 


\section{Cellular Physiology Cell Physiol Biochem 2019;52:517-531 \begin{tabular}{ll|l} 
and Biochemistry & $\begin{array}{l}\text { DOl: 10.33594/000000037 } \\
\text { Published online: 22 March 2019 }\end{array}$ & $\begin{array}{l}\text { O } 2019 \text { The Author(s). Published by } \\
\text { Cell Physiol Biochem Press GmbH\&Co. KG }\end{array}$ \\
\cline { 2 - 3 } &
\end{tabular} \\ Malhotra et al.: GAPDH—a Rapid Responder for Maintaining Iron Homeostasis in Hypoxia}

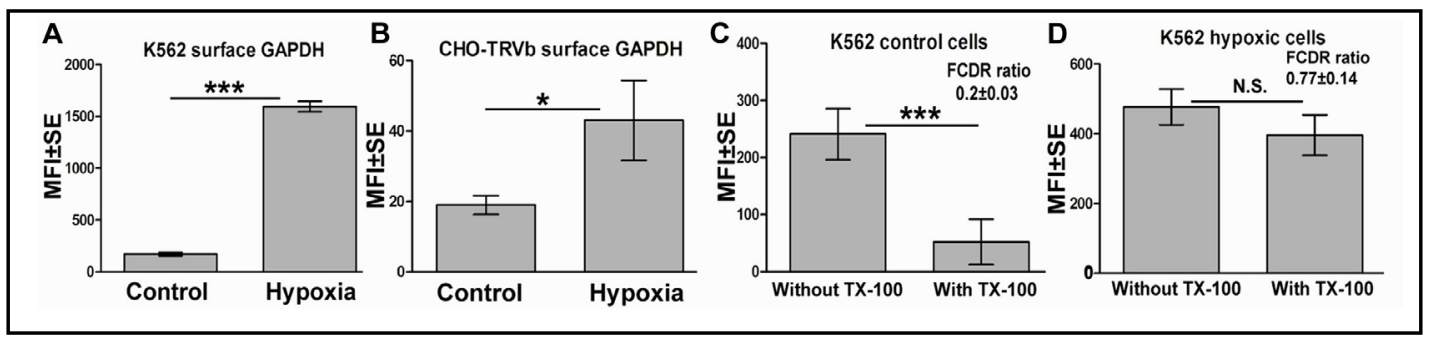

Fig. 2. Membrane recruitment of GAPDH in early stages of hypoxia. (A\&B) Cell surface GAPDH exposure is significantly increased after 24 hrs hypoxia treatment in both K562, p $<0.0001$ (A) and CHO-TRVb, p $<0.05$ (B) cells. In both cases $n=104$. (C\&D) GAPDH expressed on the plasma membrane of hypoxia treated cells is preferentially recruited to the detergent resistant membrane domain as compared to GAPDH resident on the surface of control normoxic cells. In control cells a significant decrease in surface GAPDH upon extraction with Triton X-100 was observed $(\mathrm{p}<0.0001, \mathrm{n}=104)$ indicating localization of GAPDH in non DRM portion of membrane. No such loss of GAPDH signal is observed in hypoxia treated cells suggesting migration of GAPDH molecules into the DRM region of membrane ( $>0.05, n=104)$. Representative results are shown and the entire experiment was repeated three times. In each case the FCDR ratios, were calculated using mean flowcytometry based fluorescence intensity measurements as described in materials and methods. The FCDR demonstrated significant increase upon exposure to hypoxia as compared to control cells, $\mathrm{p}<0.05$, $\mathrm{n}=3$.

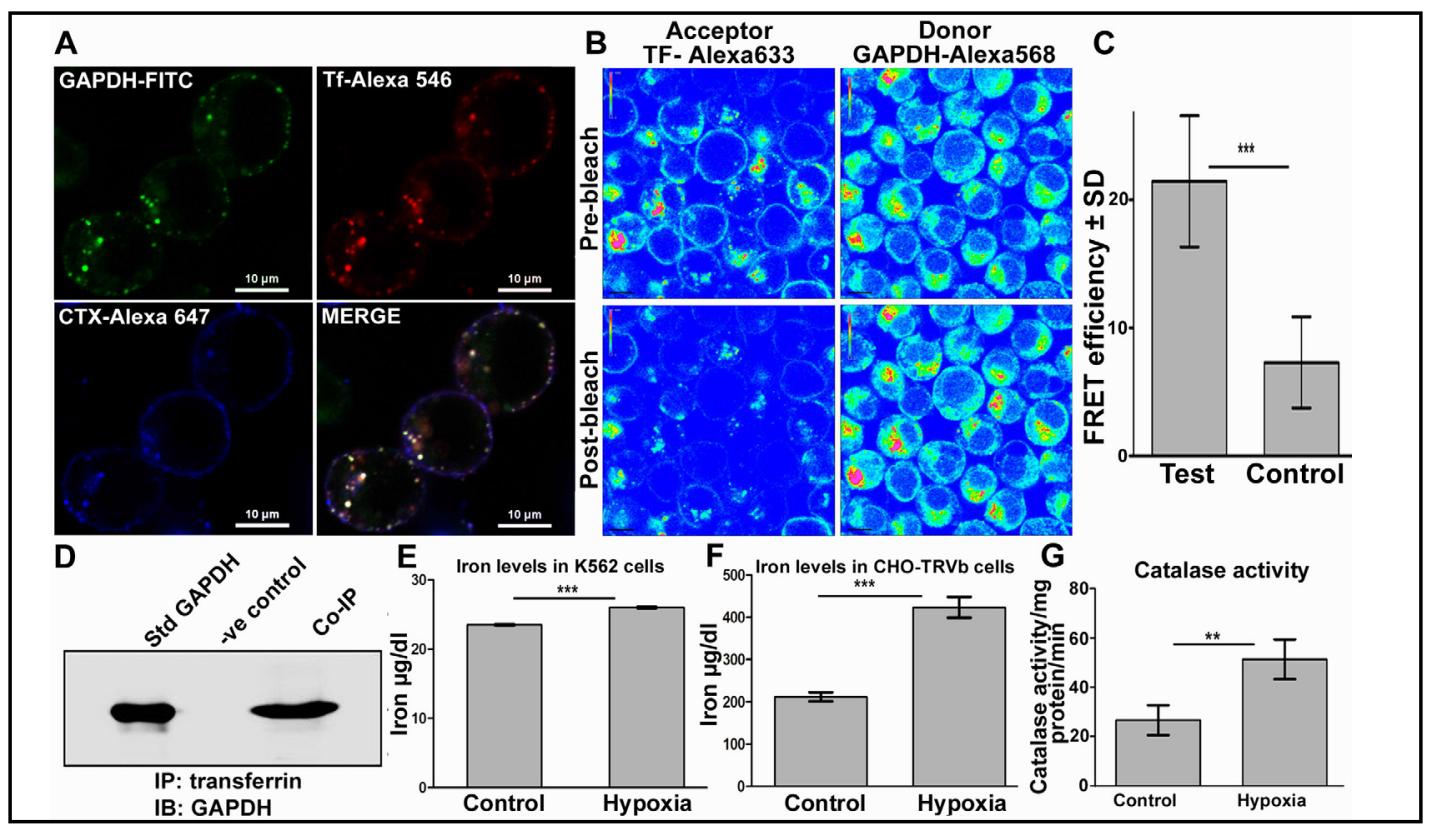

Fig. 3. Trafficking of transferrin by GAPDH under hypoxia delivers iron into cells. (A) Co-localization of Tf and GAPDH in plasma membrane rafts and endosomes of K562 cells after hypoxia exposure as observed by confocal microscopy, scale bar $10 \mu \mathrm{m}$. (B) Tf - GAPDH interaction in the endosomes of K562 cells revealed by presence of a FRET signal which is represented by an increase in fluorescence of GAPDH-Alexa 568 (donor) upon photobleaching of Tf-Alexa 633 (acceptor). Image is presented in rainbow contrast mode to highlight the increase in GAPDH signal post bleaching of acceptor (lower right panel). (C) FRET efficiency was significantly higher than control sample, where instead of, labelled Tf an unrelated donor protein goat IgG-Alexa 633 was used. $\mathrm{p}<0.0001, \mathrm{n}=58$ test and $\mathrm{n}=20$ control. (D) Co-immunoprecipitation confirms GAPDH-Tf interaction on plasma membrane of hypoxia exposed cells. (E\&F) Cellular iron levels increase after hypoxia in both K562 \& CHO-TRVb cells. For K562 cells p $<0.05$, n=3. For CHO-TRVb cells $p<0.0001$, $\mathrm{n}=3$. The increase in case of K562 cells appears to be marginal but in fact is very significant as the newly recruited GAPDH receptor has to make up for the deficit caused by decrease in TfR1 expression (seen in Fig. 1D). (G) Exposure to hypoxia enhances cellular catalase activity, $\mathrm{p}<0.05, \mathrm{n}=3$. 


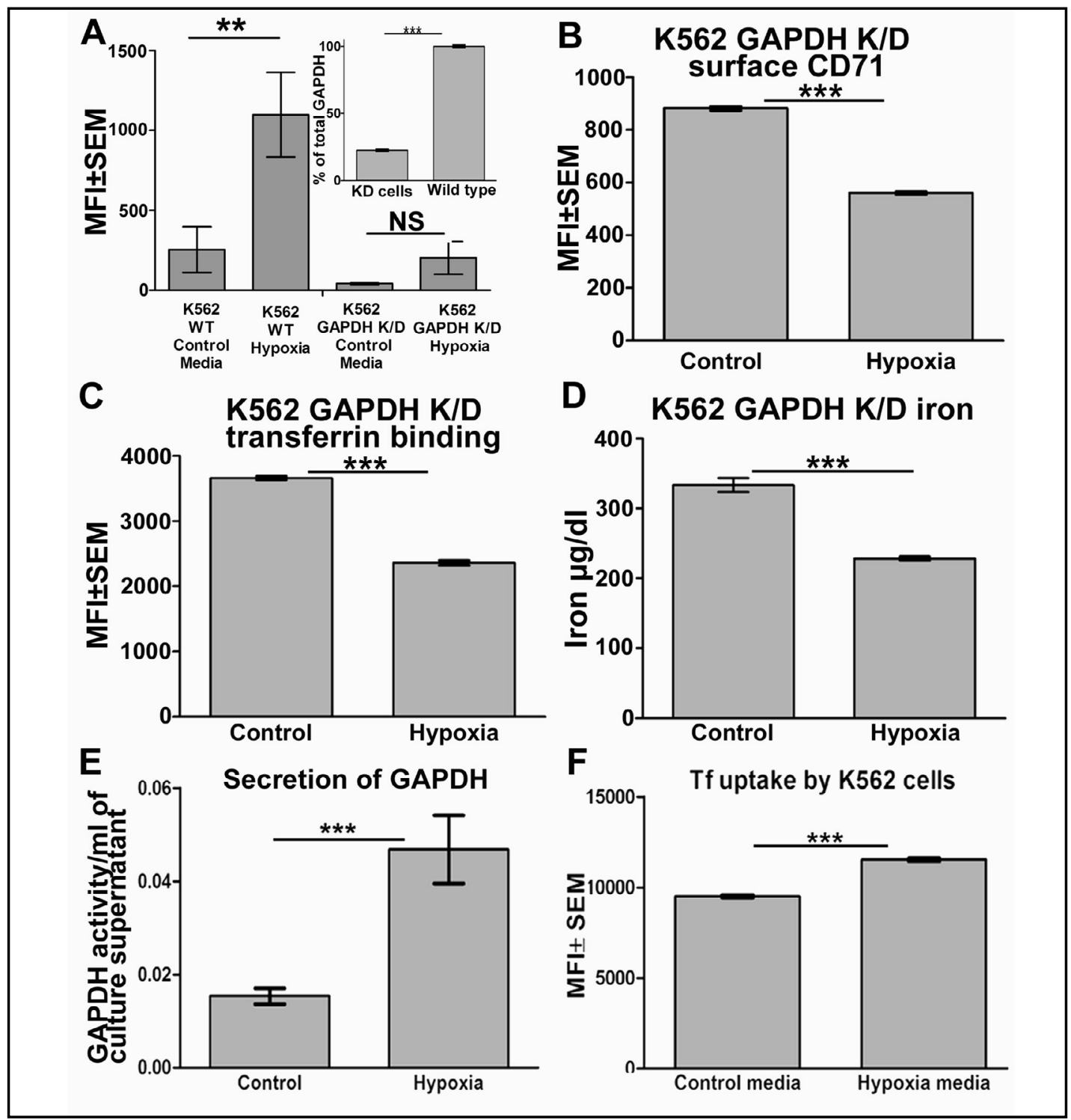

Fig. 4. Knockdown of GAPDH confirms its role in cellular acquisition of Tf and Tf bound iron during hypoxia. (A) Unlike wild type cells the GAPDH K/D cells do not show any increase in their surface GAPDH recruitment upon hypoxic exposure. K562 wild type and GAPDH knockdown cells were stained for surface GAPDH after exposure to hypoxia for $24 \mathrm{hrs}$ and analyzed by flow cytometry, control cells were maintained in regular normoxic culture conditions, ${ }^{* *} \mathrm{p}<0.001$, NS $\mathrm{p}>0.05, \mathrm{n}=10^{4}$. (A inset) Total cell GAPDH is significantly decreased in knock down cells as compared to wild type cells. $p<0.0001, n=3$. (B) As in case of wild type cells, hypoxia causes a significant decrease in CD71 expression on the surface of GAPDH K/D cells: K562 GAPDH knockdown cells were stained for surface CD71 (TfR1) after exposure to hypoxia for 24 hrs and analyzed by flow cytometry, control cells were maintained in regular normoxic culture conditions, $\mathrm{p}<0.0001, \mathrm{n}=10^{4}$. (C) Hypoxia treated GAPDH K/D cells are incapable of increasing their transferrin capture: K562 GAPDH K/D cells exposed to hypoxia for 24 hrs or maintained in normoxic culture conditions (control) and then assayed for transferrin binding by flow cytometry. A significant decrease in transferrin capture is evident, $\mathrm{p}<0.0001, \mathrm{n}=10^{4}$. (D) GAPDH knockdown cells are unable to maintain their iron uptake upon hypoxic exposure, $\mathrm{p}<0.05, \mathrm{n}=3$. (E) Hypoxia causes enhanced secretion of GAPDH. Increased levels of secreted GAPDH are present in culture supernatant of hypoxic K562 cells, $p<0.0001, n=4$. (F) Culture supernatant of hypoxic cells enriched in secreted GAPDH effects more transferrin uptake into cells. Fresh cells maintained in culture were incubated with $10 \mu \mathrm{g}$ of TF-A647Tf re-suspended in $200 \mu \mathrm{l}$ media supernatant from hypoxia exposed or control cells and evaluated for internalization of transferrin by flowcytometry, $\mathrm{p}<0.0001, \mathrm{n}=10^{4}$. 


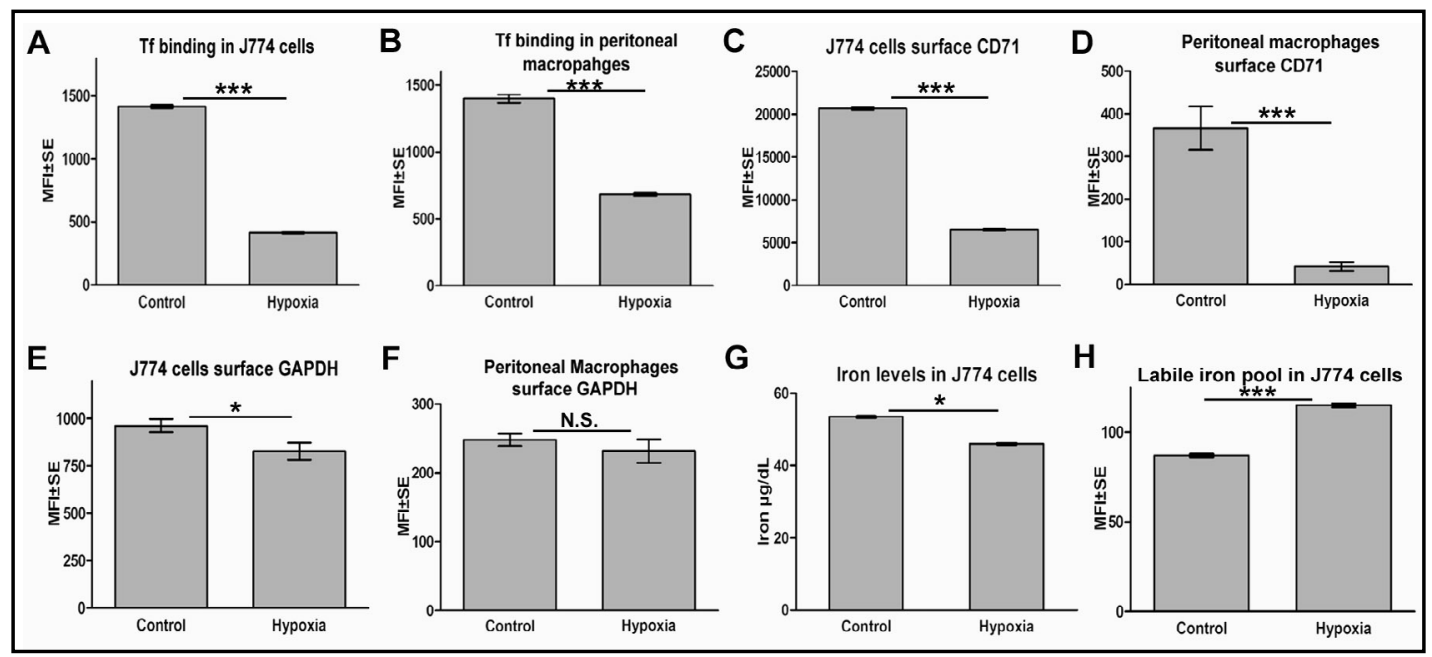

Fig. 5. Macrophages are not programmed for enhanced iron acquisition in response to hypoxia. (A\&B) In contrast to the results in K562 cells, transferrin binding is significantly decreased in macrophages subjected to hypoxia: 24 hrs hypoxia treated J774 (A) and mouse peritoneal macrophage (B) cells were assayed for transferrin binding by flow cytometry. For both cell types $p<0.0001, n=10^{4}$. (C\&D) Cell surface CD71 expression in macrophages is decreased upon exposure to hypoxia: J774 (C) and mouse peritoneal macrophages in cell culture (D) exposed to 24 hrs hypoxia were stained for surface expression of TfR1 (CD71) and evaluated by flow cytometry. Results were compared to normoxic cells. Data is presented as mean fluorescence intensity \pm SE. $p<0.0001, n=10^{4}$. (E\&F) Hypoxia exposed macrophages do not deploy GAPDH on their surface: J774 (E) and mouse peritoneal macrophage (F) cells in culture were subjected to 24 hrs of hypoxia and then evaluated for surface expression of GAPDH. No significant increase in deployment of GAPDH on plasma membrane is observed. Data is presented as mean fluorescence intensity $\pm \mathrm{SE}, \mathrm{p}<0.05$ for J774 and $p>0.05$ for peritoneal macrophages, $n=10^{4}$. (G) Decreased cellular iron levels in hypoxic J774 cells as hypoxia induces macrophage cells to release iron, $p<0.05, n=3$. (H) Calcein quenching assay reveals a depleted labile iron pool. As presence of iron quenches calcein florescence the observed increase in signal from hypoxic J774 cells indicates a depleted labile iron pool. Data is presented as mean fluorescence intensity \pm SE, $\mathrm{p}<0.0001, \mathrm{n}=10^{4}$.

\section{Macrophages exhibit a disparate role in terms of GAPDH expression during hypoxia}

Macrophages play a key role in iron metabolism, phagocytosing effete RBCs in the spleen to recover and recycle iron back into circulation for the synthesis of new hemoglobin intended for maturing erythroid cells in bone marrow [43]. To successfully fulfil this role under hypoxia, macrophages are required to function as dispensers of stored iron rather than as consumers of the metal. In agreement with this phenomenon, we observed decreased binding of Tf to hypoxia-exposed macrophage cells (Fig. 5A \& B). The reason for this was clear when we examined the expression of CD71 and GAPDH on the surface of these cells and found that while CD71 expression was significantly decreased (Fig. 5C \& D), the GAPDH levels were either slightly decreased or remained unaltered (Fig. 5E \& F). We evaluated the total iron status as well as the labile iron pool in hypoxia-treated macrophage cells and found both to be decreased (Fig. 5G \& H). These contrary results in macrophages (compared to K562 cells) indicate that the enhancement of GAPDH upon hypoxia is a cell-specific response to meet the tissue-specific requirements of iron. As iron-retaining cells, macrophages are tuned for the release of sequestered iron during hypoxic stress so as to meet the enhanced requirements of bone marrow and other principal iron-utilizing tissues. It has been reported previously that hypoxia enhances the release of iron from U937 macrophages [44]. Another study showed that exposure of human subjects to high altitude hypoxia (especially in the early phase) results in a decrease in TfR1 and ferritin coupled with an upregulation of ferroportin in muscle tissue (which is also an iron-storage tissue as are macrophages) [45]. 


\section{Cellular Physiology Cell Physiol Biochem 2019;52:517-531 \\ \begin{tabular}{l|l} 
DDI: 10.33594/000000037 & O 2019 The Author(s). Published by \\
and Biochemistry Published online: 22 March 2019 & Cell Physio Biochem Press GmbH\&Co. KG
\end{tabular} \\ Malhotra et al.: GAPDH—a Rapid Responder for Maintaining Iron Homeostasis in \\ Hypoxia}

\section{Discussion}

Previously, we established that upon sensing an iron starvation stress, cells rapidly deploy GAPDH to membrane raft regions and also secrete it so as to capture and internalize transferrin and restore intracellular iron levels. The detailed biochemical and biophysical characteristics regarding the interaction of this GAPDH with transferrin and the subsequent endocytotic machinery in replenishing cellular iron have been described earlier [17, 24]. Our present study highlights the role of GAPDH in conditions of cellular hypoxic stress, where it also functions as a primary rapid-response molecule which is quickly deployed to the cell membrane raft domain so as to maintain and bolster vital iron supplies before more evolved molecules can be synthesized and recruited to the membrane after a substantial time lag. GAPDH is an extremely multifunctional protein with which novel functions are regularly associated [46-48]. Present in very high copy numbers, its extreme versatility renders GAPDH akin to a cell's "Swiss Army Knife." It comprises a vast pool of readily available and adaptable molecules that can be called upon to perform diverse and essential functions at short notice before specialized molecules that have evolved to perform singular functions can be expressed and deployed in sufficient quantities. In addition to a housekeeping role in glycolysis, GAPDH assists in maintaining the homeostatic balance of the crucial micronutrient iron. This highly pleiotropic molecule has been well conserved in sequence and structure from prokaryotes to higher life forms. Previous studies involving prokaryotic and metazoan cells have revealed a conserved role for GAPDH in the maintenance of cellular iron homeostasis [49]. This led us to postulate that GAPDH may constitute a primeval iron acquisition machinery that has been retained throughout evolution $[21,26]$. The occurrence of cellular hypoxia, the high demand for iron as a cofactor for enzymes involved in cell replication, and an increase in transferrin receptors and enhanced anaerobic respiration via the upregulation of glycolytic enzymes are common properties of many tumors. As such, our findings may be relevant to physiological conditions wherein elevated levels of GAPDH have been associated with poor prognosis and tumor aggressiveness.

\section{Acknowledgements}

Mr. Anil Theophilus and Mr. Randeep Sharma are acknowledged for their technical assistance. A.S.C., A.D. and A.P. received fellowships from DBT, N.S., K.S., P.J. and M.K. received financial support from CSIR, while H.M. and S.C. received fellowships from UGC. Financial support from CSIR, DBT, and DST is acknowledged. All animal handling protocols including those for obtaining primary cells were as per the Institute of Microbial Technology Experimental Animal Facility protocols. The procedures were approved by the statutory Institutional Animal Ethics Committee and were performed strictly as per the guidelines of the Committee for the Purpose of Control and Supervision of Experiments on Animals (CPCEA), Ministry of Environment, Forests and Climate Change, Government of India.

\section{Disclosure Statement}

The authors have no conflicts of interest to report. 


\section{Cellular Physiology Cell Physiol Biochem 2019;52:517-531 \begin{tabular}{c|l}
\cline { 2 - 3 } DOI: 10.33594/000000037 & (c) 2019 The Author(s). Published by
\end{tabular} and Biochemistry Published online: 22 March $2019 \quad$ Cell Physiol Biochem Press GmbH\&Co. KG \\ Malhotra et al.: GAPDH—a Rapid Responder for Maintaining Iron Homeostasis in Hypoxia}

\section{References}

- 1 Chepelev NL, Willmore WG: Regulation of iron pathways in response to hypoxia. Free Radic Biol Med 2011;50:645-666.

- 2 Cavill I: Erythropoiesis and iron. Best Pract Res Clin Haematol 2002;15:399-409.

- 3 Kawabata H, Yang R, Hirama T, Vuong PT, Kawano S, Gombart AF, Koeffler HP: Molecular cloning of transferrin receptor 2 A new member of the transferrin receptor-like family. J Biol Chem 1999;274:2082620832.

- 4 West AP, Bennett MJ, Sellers VM, Andrews NC, Enns CA, Bjorkman PJ: Comparison of the interactions of transferrin receptor and transferrin receptor 2 with transferrin and the hereditary hemochromatosis protein HFE. J Biol Chem 2000;275:38135-38138.

- 5 Toth I, Yuan L, Rogers JT, Boyce H, Bridges KR: Hypoxia alters iron-regulatory protein-1 binding capacity and modulates cellular iron homeostasis in human hepatoma and erythroleukemia cells. J Biol Chem 1999;274:4467-4473.

- 6 Piperno A, Galimberti S, Mariani R, Pelucchi S, Ravasi G, Lombardi C, Bilo G, Revera M, Giuliano A, Faini A: Modulation of hepcidin production during hypoxia-induced erythropoiesis in humans in vivo: data from the HIGHCARE project. Blood 2011;117:2953-2959.

- 7 Zhang Y, Singh I, Krzyzanski W: Dynamics of erythropoietic markers in response to hypoxia in rats. J Blood Disord Transfus 2014;5:197.

- 8 Eckardt K-U, Boutellier U, Kurtz A, Schopen M, Koller EA, Bauer C: Rate of erythropoietin formation in humans in response to acute hypobaric hypoxia. J Appl Physiol 1989;66:1785-1788.

- 9 Clanton TL: Hypoxia-induced reactive oxygen species formation in skeletal muscle. J Appl Physiol 2007;102:2379-2388.

- 10 Chandel NS, McClintock DS, Feliciano CE, Wood TM, Melendez JA, Rodriguez AM, Schumacker PT: Reactive Oxygen Species Generated at Mitochondrial Complex III Stabilize Hypoxia-inducible Factor-1 $\alpha$ during Hypoxia: a mechanism of 02 sensing. J Biol Chem 2000;275:25130-25138.

11 Lobo V, Patil A, Phatak A, Chandra N: Free radicals, antioxidants and functional foods: Impact on human health. Pharmacogn Rev 2010;4:118-126.

- 12 Rauchová H, Vokurkova M, Koudelova J: Developmental changes of erythrocyte catalase activity in rats exposed to acute hypoxia. Physiol Res 2005;54:527-532.

- 13 Turrens JF: Mitochondrial formation of reactive oxygen species. J Physiol 2003;552:335-344.

14 Lok CN, Ponka P: Identification of a hypoxia response element in the transferrin receptor gene. J Biol Chem 1999;274:24147-24152.

15 Tao J, Liu Yq, Li Y, Peng Jl, Li L, Liu J, Shen X, Shen Gx, Tu Yt: Hypoxia: dual effect on the expression of transferrin receptor in human melanoma A375 cell line. Exp Dermatol 2007;16:899-904.

16 Zhang FL, Shen GM, Liu XL, Wang F, Zhao YZ, Zhang JW: Hypoxia-inducible factor 1-mediated human GATA1 induction promotes erythroid differentiation under hypoxic conditions. J Cell Mol Med 2012;16:1889-1899.

- 17 Kumar S, Sheokand N, Mhadeshwar MA, Raje CI, Raje M: Characterization of glyceraldehyde-3-phosphate dehydrogenase as a novel transferrin receptor. Int J Biochem Cell Biol 2012;44:189-199.

- 18 Modun B, Evans RW, Joannou CL, Williams P: Receptor-mediated recognition and uptake of iron from human transferrin by Staphylococcus aureus and Staphylococcus epidermidis. Infect Immun 1998;66:3591-3596.

19 Rawat P, Kumar S, Sheokand N, Raje CI, Raje M: The multifunctional glycolytic protein glyceraldehyde3-phosphate dehydrogenase (GAPDH) is a novel macrophage lactoferrin receptor. Biochem Cell Biol 2012;90:329-338.

20 Henderson B: Moonlighting Proteins: Novel Virulence Factors in Bacterial Infections. Hoboken, New Jersey, John Wiley \& Sons, Inc., 2017.

- 21 Boradia VM, Raje M, Raje CI: Protein moonlighting in iron metabolism: glyceraldehyde-3-phosphate dehydrogenase (GAPDH). Biochem Soc Trans 2014;42:1796-1801.

- 22 Malhotra H, Patidar A, Boradia VM, Kumar R, Nimbalkar RD, Kumar A, Gani Z, Kaur R, Garg P, Raje M, Raje CI: Mycobacterium tuberculosis Glyceraldehyde-3-Phosphate Dehydrogenase (GAPDH) Functions as a Receptor for Human Lactoferrin. Front Cell Infect Microbiol 2017;7:245. 


\section{Cellular Physiology Cell Physiol Biochem 2019;52:517-531 \begin{tabular}{l|l|l}
\hline DOI: 10.33594/000000037 & (c) 2019 The Author(s). Published by
\end{tabular} and BiOChemistry Published online: 22 March 2019 Cell Physiol Biochem Press GmbH\&Co. KG \\ Hypoxia}

23 Chauhan AS, Rawat P, Malhotra H, Sheokand N, Kumar M, Patidar A, Chaudhary S, Jakhar P, Raje CI, Raje M: Secreted multifunctional Glyceraldehyde-3-phosphate dehydrogenase sequesters lactoferrin and iron into cells via a non-canonical pathway. Sci Rep 2015;5:18465.

24 Sheokand N, Kumar S, Malhotra H, Tillu V, Raje CI, Raje M: Secreted glyceraldehye-3-phosphate dehydrogenase is a multifunctional autocrine transferrin receptor for cellular iron acquisition. Biochim Biophys Acta Gen Subj 2013;1830:3816-3827.

- 25 McGraw TE, Greenfield L, Maxfield FR: Functional expression of the human transferrin receptor cDNA in Chinese hamster ovary cells deficient in endogenous transferrin receptor. J Cell Biol 1987;105:207-214.

- 26 Raje CI, Kumar S, Harle A, Nanda JS, Raje M: The macrophage cell surface glyceraldehyde-3-phosphate dehydrogenase is a novel transferrin receptor. J Biol Chem 2007;282:3252-3261.

- 27 Kato J, Kobune M, Ohkubo S, Fujikawa K, Tanaka M, Takimoto R, Takada K, Takahari D, Kawano Y, Kohgo Y: Iron/IRP-1-dependent regulation of mRNA expression for transferrin receptor, DMT1 and ferritin during human erythroid differentiation. Exp Hematol 2007;35:879-887.

28 Roehrich ME, Mooser V, Lenain V, Herz J, Nimpf J, Azhar S, Bideau M, Capponi A, Nicod P, Haefliger JA: Insulin-secreting $\beta$-cell dysfunction induced by human lipoproteins. J Biol Chem 2003;278:18368-18375.

29 Livak KJ: Analysis of relative gene expression data using real-time quantitative PCR and the $2(\Delta \Delta \mathrm{C} /(\mathrm{T}))$ methods. Methods 2001;25:402-408.

- 30 Chauhan AS, Kumar M, Chaudhary S, Patidar A, Dhiman A, Sheokand N, Malhotra H, Raje CI, Raje M: Moonlighting glycolytic protein glyceraldehyde-3-phosphate dehydrogenase (GAPDH): an evolutionarily conserved plasminogen receptor on mammalian cells. FASEB J 2017;31:2638-2648.

- 31 Gombos I, Bacsó Z, Detre C, Nagy H, Goda K, Andrásfalvy M, Szabó G, Matkó J: Cholesterol sensitivity of detergent resistance: a rapid flow cytometric test for detecting constitutive or induced raft association of membrane proteins. Cytometry Part A 2004;61:117-126.

- 32 Boradia VM, Malhotra H, Thakkar JS, Tillu VA, Vuppala B, Patil P, Sheokand N, Sharma P, Chauhan AS, Raje M, Raje CI: Mycobacterium tuberculosis acquires iron by cell-surface sequestration and internalization of human holo-transferrin. Nat Commun 2014;5:4730.

33 Sheokand N, Malhotra H, Chauhan AS, Kumar M, Chaudhary S, Patidar A, Boradia VM, Raje CI, Raje M: Reverse overshot water-wheel retroendocytosis of Apo Transferrin extrudes cellular iron. J Cell Sci 2016;129:843-853.

34 Anderson MD, Prasad TK, Stewart CR: Changes in isozyme profiles of catalase, peroxidase, and glutathione reductase during acclimation to chilling in mesocotyls of maize seedlings. Plant Physiol 1995;109:12471257.

- 35 Zhong H, Simons JW: Direct comparison of GAPDH, $\beta$-actin, cyclophilin, and 28S rRNA as internal standards for quantifying RNA levels under hypoxia. Biochem Biophys Res Commun 1999;259:523-526.

- 36 Yamaji R, Fujita K, Takahashi S, Yoneda H, Nagao K, Masuda W, Naito M, Tsuruo T, Miyatake K, Inui H: Hypoxia up-regulates glyceraldehyde-3-phosphate dehydrogenase in mouse brain capillary endothelial cells: involvement of Na+/Ca 2+ exchanger. Biochim Biophys Acta Mol Cell Res 2003;1593:269-276.

\ 37 Alpay Z, Özgönenel M, Savaşan S, Buck S, Saed GM, Diamond MP: Altered in vitro immune response to hypoxia-treated normal peritoneal fibroblasts. Fertil Steril 2007;87:426-429.

- 38 Goralska M, Fleisher LN, McGahan MC: Hypoxia induced changes in expression of proteins involved in iron uptake and storage in cultured lens epithelial cells. Exp Eye Res 2014;125:135-141.

39 Schneider BD, Leibold EA: Effects of iron regulatory protein regulation on iron homeostasis during hypoxia. Blood 2003;102:3404-3411.

40 Calzolari A, Finisguerra V, Oliviero I, Deaglio S, Mariani G, Malavasi F, Testa U: Regulation of transferrin receptor 2 in human cancer cell lines. Blood Cells Mol Dis 2009;42:5-13.

41 Calzolari A, Deaglio S, Sposi NM, Petrucci E, Morsilli O, Gabbianelli M, Malavasi F, Peschle C, Testa U: Transferrin receptor 2 protein is not expressed in normal erythroid cells. Biochem J 2004;381:629-634.

- 42 Sheokand N, Malhotra H, Kumar S, Tillu VA, Chauhan AS, Raje CI, Raje M: Moonlighting cell-surface GAPDH recruits apotransferrin to effect iron egress from mammalian cells. J Cell Sci 2014;127:4279-4291.

- 43 Ganz T: Macrophages and systemic iron homeostasis. J Innate Immun 2012;4:446-453.

- 44 Sarkar J, Seshadri V, Tripoulas NA, Ketterer ME, Fox PL: Role of ceruloplasmin in macrophage iron efflux during hypoxia. J Biol Chem 2003;278:44018-44024. 


\section{Cellular Physiology Cell Physiol Biochem 2019;52:517-531}

\begin{tabular}{ll|l} 
and Biochemistry & DOl: 10.33594/000000037 & Published online: 22 March 2019 The Author(s). Published by \\
Cell Physiol Biochem Press GmbH\&Co. KG
\end{tabular}

Malhotra et al.: GAPDH—a Rapid Responder for Maintaining Iron Homeostasis in

Hypoxia

- 45 Robach P, Cairo G, Gelfi C, Bernuzzi F, Pilegaard H, Vigano A, Santambrogio P, Cerretelli P, Calbet JA, Moutereau S: Strong iron demand during hypoxia-induced erythropoiesis is associated with downregulation of iron-related proteins and myoglobin in human skeletal muscle. Blood 2007;109:4724-4731.

- 46 Sirover MA: On the functional diversity of glyceraldehyde-3-phosphate dehydrogenase: biochemical mechanisms and regulatory control. Biochim Biophys Acta Gen Subj 2011;1810:741-751.

47 Seidler NW: GAPDH: Biological Properties and Diversity: Biological Properties and Diversity, Springer, 2012.

- 48 White MR, Garcin ED: D-Glyceraldehyde-3-Phosphate Dehydrogenase Structure and Function, in Harris JR, Marles-Wright J (eds): Macromolecular Protein Complexes: Structure and Function. Cham, Springer International Publishing, 2017, pp 413-453.

49 Boradia VM, Raje M, Raje CI: Mycobacterium tuberculosis Cell-Surface GAPDH Functions as a Transferrin Receptor, in Henderson B (ed): Moonlighting Proteins: Novel Virulence Factors in Bacterial Infections. Hoboken, New Jersey, John Wiley \& Sons, Inc., 2017, pp 205-224. 